\title{
Tratamiento quirúrgico de las metástasis hepáticas de cáncer colorrectal. De las resecciones locales al trasplante hepático
}

\author{
Nicolás Jarufe C. ${ }^{1}$, Gabriela Ochoa S. ${ }^{1}$, Martin Dib M. ${ }^{1}$, \\ Jorge Martínez C. ${ }^{1}$ y Eduardo Briceño V. ${ }^{1}$
}

\section{Surgical treatment of hepatic metastases in colorectal cancer. From local resections to liver transplantation}

Colorectal cancer is one of the leading causes of cancer death worldwide. A significant proportion of cases develop liver metastases. Thanks to diagnostic advances and oncologic treatments, survival has been increasing, however, it is essential to achieve complete surgical resection of primary and metastatic lesions with negative microscopic margins (R0). There are many procedures and techniques designed for this purpose such as neoadjuvant chemotherapy, portal embolization, stage surgery, etc. Despite this, there are non-resectable cases due to bilobar, multisegmental and/or hepatic involvement of arterial, portal or venous vessels that, in case of resection, do not allow the maintenance of functional remnant liver compatible with the patient's life. Liver transplantation emerges as a radical alternative for the treatment of unresectable cases. Given the shortage of donors and mortality on the national waiting list, it is not acceptable to occupy donor pool livers for pathologies with expanded criteria such as colorectal metastases. However, with the resource of the living donor from adult to adult, today it is possible to indicate transplantation in selected cases, which meet all established criteria.

Key words: liver metastases; colorectal; transplant.

\section{Resumen}

El cáncer colorrectal es de las principales causas de muerte por cáncer a nivel mundial. Una proporción importante de los casos desarrolla metástasis hepáticas. Gracias a los avances diagnósticos y tratamientos oncológicos, la sobrevida ha ido en aumento, sin embargo, para ello es fundamental lograr la resección quirúrgica completa de las lesiones primarias y metastásicas con márgenes microscópicos negativos (R0). Existen numerosos procedimientos y técnicas diseñadas para este objetivo como la quimioterapia neoadyuvante, embolización portal, cirugía en etapas, etc. A pesar de ello, hay casos no resecables por compromiso hepático bilobar, multisegmentario y/o compromiso de vasos arteriales, portales o venosos que en caso de resección, no permiten mantener hígado remanente funcional compatible con la vida del paciente. El trasplante hepático surge como alternativa radical para el tratamiento de casos no resecables. Dado la escasez de donantes y mortalidad en la lista de espera nacional, no es aceptable ocupar hígados del pool de donantes para patologías con criterios expandidos como metástasis colorrectales. Sin embargo, con el recurso del donante vivo de adulto a adulto, hoy en día es posible indicar trasplante en casos seleccionados, que cumplan con todos los criterios establecidos.

Palabras clave: metástasis hepáticas; colorrectal; trasplante.
Departamento de Cirugía Digestiva. Pontificia Universidad Católica de Chile. Santiago, Chile.

Recibido el 29 de agosto de 2019 y aceptado para publicación el

Correspondencia a: Dr. Nicolás Jarufe C. njarufe@clc.cl 


\section{Introducción}

El cáncer colorrectal $(\mathrm{CCR})$ es la tercera neoplasia maligna más frecuente del mundo ${ }^{1}$, con una incidencia de 1,8 millones casos nuevos el año 2018 y es la segunda causa más frecuente de muerte por cáncer a nivel mundial ${ }^{2}$. En Chile, es el quinto cáncer más frecuente y tiene una mortalidad de $10,3 / 100.000$ hab (2015), cifras que van en aumento en la última década ${ }^{3}$.

De los pacientes con CCR, el 25\% tiene metástasis hepáticas $(\mathrm{MH})$ al momento del diagnóstico, $50 \%$ las desarrollarán en el curso de su enfermedad y $33 \%$ sólo tendrá metástasis en el hígado. La sobrevida natural de los pacientes con $\mathrm{MH}$ es de 6 a 12 meses con una sobrevida a 5 años de $10 \%$, lo cual aumenta a 24-32 meses cuando son sometidos a $\mathrm{QMT}^{4,5}$. Sin embargo, la resección de $\mathrm{MH}$ ofrece la mayor probabilidad de curación para pacientes en etapa IV, logrando actualmente sobrevidas a 5 años de $40 \%-45 \%$, similares a los pacientes con CCR etapa III. Lamentablemente, sólo $20 \%$ de los pacientes con MHCCR se consideran resecables ${ }^{4,8}$.

La resección en $\mathrm{MH}$ es una técnica segura, con mortalidad perioperatoria de $2 \%$ y morbilidad en continuo descenso siendo menor a $20-30 \%$ hoy en día. Estas cifras han permitido ampliar las estrategias de manera de lograr finalmente el R0 (márgenes microscópicos negativos) que ha demostrado ser lo más efectivo en términos de sobrevida a largo plazo $^{8}$.

\section{Definición actual de resecabilidad}

Los criterios de resecabilidad han tenido una evolución continua. En 1986 se estableció por Ekberg como resecables aquellos pacientes con menos de 4 lesiones, sin evidencia de enfermedad extrahepática y la posibilidad de obtener márgenes mayores a $1 \mathrm{~cm}^{9}$.

El consenso de expertos realizado el año 2006 en San Francisco, California, estableció que para considerar resecables las MHCCR, el remanente hepático debía cumplir con: tener al menos 2 segmentos hepáticos en continuidad, irrigación portal, arterial y drenaje biliar (o al intestino) y corresponder al menos al 20-25\% del volumen hepático (o $40 \%$ en caso de QMT preoperatoria u otro daño hepático), siendo fundamental lograr una resección R $0^{11}$.

Un nuevo consenso de expertos HPB el año 2012 determinó que para definir resecabilidad había que tener en consideración dos aspectos: oncológicos y técnicos. La evaluación oncológica debe estar basa- da en la biología del tumor (carga tumoral, enfermedad extrahepática, respuesta a terapia neoadyuvante, perfil de mutaciones). La evaluación técnica debe estar referida a obtener márgenes negativos preservando un remanente hepático futuro con adecuada irrigación y drenaje vascular y biliar ${ }^{4,9}$.

Con el advenimiento de terapias sistémicas más efectivas, terapias biológicas específicas, desarrollo de nuevas técnicas quirúrgicas, mayor tecnología y mejoras en el manejo perioperatorio, se han ampliado los criterios de resecabilidad de manera de lograr el ansiado R0 $0^{4,5,9}$.

Los criterios actuales aceptados para resección hepática incluyen cualquier número de tumores, de cualquier localización en el hígado, con enfermedad extrahepática estable o resecable, remanente hepático futuro adecuado, compromiso venoso susceptible a resección o incluso reconstrucción, siempre manteniendo el objetivo fundamental de lograr márgenes microscópicos negativos ${ }^{4}$.

\section{Remanente hepático futuro (RHF)}

$\mathrm{Al}$ expandir los criterios de resección de $\mathrm{MH}-$ CRC, es necesario considerar el RHF con el fin de evitar la insuficiencia hepática postquirúrgica. Cuando el remanente es menor a $25 \%$ existe mayor riesgo de morbimortalidad en pacientes con función hepática normal. El uso de quimioterapia neoadyuvante produce toxicididad hepática, por lo que en estos pacientes se requiere un RHF de al menos $30 \%$. Los hígados con cirrosis suponen un grado de disfunción mayor, por lo cual el remanente en ellos debe ser de al menos $40 \% 0^{9,12}$.

\section{Márgenes oncológicos}

Históricamente el margen oncológico para lograr R0 debía ser mayor a $1 \mathrm{~cm}$. Con los estudios de Pawlik el año 2005 (n: 557 pacientes) y E. Sadot 2015 (n: 2.368 pacientes), se objetivó que, si bien márgenes inferiores a $1 \mathrm{~mm}$ tenían peor sobrevida, márgenes subcentimétricos mayores a $1 \mathrm{~mm}$ no tenían impacto negativo en ésta ni en el riesgo de recurrencia ${ }^{14,15}$.

Actualmente, el objetivo terapéutico es lograr márgenes sobre $1 \mathrm{~mm}^{9}$.

\section{Estudio de imágenes}

Tiene como fin la etapificación del paciente. Debe definir el número, tamaño y distribución lobar/ segmentaria de las metástasis, determinar la resecabilidad quirúrgica fundamentalmente por la relación con las estructuras cruciales del hígado (venas 
suprahepáticas, vena porta, arteria hepática y vías biliares), identificar la enfermedad concomitante del parénquima, definir el RHF y caracterizar las lesiones de acuerdo a su respuesta a la quimioterapia ${ }^{5,16}$.

Para la planificación de la resección de lesiones hepáticas, se utilizan:

- TC: En la etapificación inicial del tumor y la enfermedad extrahepática (de tórax, abdomen y pelvis). También es útil en la detección de recurrencia de enfermedad.

- RNM: En términos de planificación de la resección tiene similar sensibilidad que el TC (94\% vs 91\%), pero tiene mayor sensibilidad en la detección de $\mathrm{MH}(91 \%$ vs $82 \%)$, especialmente en lesiones subcentimétricas y en la evaluación del hígado postquimioterapia ${ }^{9}$.

- PET-CT: Su rol fundamental es en la detección de enfermedad extrahepática. Su utilidad en la planificación de pacientes con lesiones potencialmente resecables ha sido controversial. El estudio randomizado de Serrano publicado el año 2018 demostró que no había cambios en la sobrevida global ni libre de enfermedad frente al uso preoperatorio de PET-CT ${ }^{17}$. La NCCN no lo recomienda de uso rutinario, debido a que la QMT preoperatoria puede reducir su sensibilidad.

- Volumetría 3D (TC): Es indispensable para evaluar el RHF, y disminuir la posibilidad de falla hepática preoperatoria, especialmente en hepatectomías mayores ${ }^{4,9}$.

\section{Estrategia en metástasis hepáticas sincrónicas vs metacrónicas}

Según el momento de presentación en relación con el tumor primario las podemos dividir en metacrónicas y sincrónicas, estando asociadas estas últimas a peor pronóstico.

Las MH metacrónicas no difieren técnicamente del manejo de otras resecciones hepáticas.

En MH sincrónicas existe controversia con respecto al orden del manejo: tumor primario, $\mathrm{MH}$ o neoadyuvancia. Primero se debe considerar si tumor primario es sintomático (sangrado, obstrucción, perforación), ya que estos pacientes deben ser manejados con "abordaje clásico" (primero el colon) y posteriormente las $\mathrm{MH}$, por el riesgo de complicación ${ }^{5}$.

Cuando el tumor primario es asintomático, se debe definir si se realizará la resección de colon e hígado en un tiempo, o en diferido. La posibilidad de abordaje en un tiempo está limitada por la necesidad de una cirugía compleja o extensa de alguna de las lesiones, ya que esto se asocia a mayor morbilidad, por lo cual no es recomendado 5 .

En el enfrentamiento diferido, se debe tener en cuenta para la decisión la carga tumoral y la posibilidad de lograr R0, existiendo las opciones de abordaje clásico o reverso (hígado primero). En enfermedad hepática extensa probablemente no se logre R0 en una sola cirugía, por lo que estos pacientes se beneficiarían del manejo clásico para poder completar esquemas de neoadyuvancia, y viceversa en caso de extenso compromiso del tumor primario con linfonodos positivos asociados ${ }^{5}$.

\section{Resecciones anatómicas $(R A)$ vs preservación de parénquima (PP)}

Inicialmente se consideraba que las RA eran la regla en patología neoplásica. Sin embargo, diversos estudios no han demostrado diferencias en sobrevida global, sobrevida libre de enfermedad o márgenes comprometidos entre RA y PP. La PP (resecciones económicas) han demostrado menor tasa de morbilidad global, menor riesgo de falla hepática aguda y menor necesidad de cuidados intensivos postoperatorios. Además, tienen la ventaja de favorecer las re-resecciones en caso de recurrencia, siendo actualmente el estándar ${ }^{18,19}$.

\section{Desaparición de metástasis \\ (Missing-metastasis)}

Existe un grupo de pacientes que posterior al tratamiento con quimioterapia presentan respuesta radiológica completa (5-38\% de los casos). La habilidad para encontrar estas metástasis en el intraoperatorio es modesta (hasta 40\%), a pesar del uso de ecografía intraoperatoria. Se ha logrado aumentar la tasa de detección con agentes de contraste específicos de hepatocitos, pero sigue siendo baja. La problemática que esto supone es conocer el grado de concordancia entre la respuesta imagenológica completa y la respuesta patológica completa, y su duración en el tiempo. Se ha reportado que $25-45 \%$ de los pacientes con respuesta imagenológica completa tienen enfermedad macroscópica residual al momento de la resección. Actualmente, existe controversia respecto a resecar o no el territorio donde se encontraban las metástasis antes de desaparecer. Si bien la recurrencia de estas es elevada, técnicamente es difícil precisar el sitio de resección y, por lo tanto, podría ser más recomendable la cirugía solamente en casos de reaparición. Alternativamente, previo a recibir quimioterapia se podrían marcar con coils especiales percutáneos, aquellas con alto riesgo de desaparecer como las metástasis pequeñas $(<1 \mathrm{~cm})$, múltiples y bilobares ${ }^{9}$. 


\section{Re-resecciones}

El $60-70 \%$ de los pacientes con MHCRC operadas van a recurrir. El $30 \%$ lo hará en el hígado. La re-resección es una cirugía técnicamente más demandante, pero con una morbimortalidad similar a la primera resección y que alcanza sobrevida a 5 años de $30 \%$, por lo que es una opción válida y segura de tratamiento.

\section{Resecciones laparoscópicas}

Los primeros reportes de resecciones laparoscópicas hepáticas (RLH) mostraban hasta 10\% de mortalidad, por lo cual durante el desarrollo de la técnica ha sido fundamental la selección adecuada de pacientes. Actualmente, centros especializados reportan mortalidad de $2 \%$ con tasas de conversión de 5\%. La curva de aprendizaje es larga y se considera que es de 60 casos previo a adquirir la competencia ${ }^{19}$.

En general, existen condiciones favorables para RLH tanto del tumor (menores de $5 \mathrm{~cm}$, localizaciones periféricas) como del tipo de resección (ej: segmentectomías laterales izquierdas). Los criterios de Iwate clasifican a los pacientes según tamaño tumoral, localización, proximidad a vasos mayores, extensión de la resección, incorporación de técnica mano asistida y función hepática previa y les asignan un índice que predice dificultad ${ }^{19}$.

En el estudio randomizado OSLO-COMET (2018), que incluyó 280 pacientes, las RLH tuvieron una menor tasa de morbilidad que las resecciones abiertas (hepatectomías menores). No hubo diferencias en compromiso de los márgenes, tiempo operatorio, necesidad de transfusiones. Sin diferencia significativa en mortalidad a 90 días $^{21}$.

RLH en hepatectomías mayores, deben ser realizadas en centros especializados, donde se ha desarrollado la técnica de forma segura ${ }^{4,19}$.

\section{Cirugía robótica en resecciones hepáticas}

Aún no hay suficiente evidencia para apoyar la cirugía robótica en resecciones por MHCCR. En general se supone que pudiese tener ventaja en el abordaje de tumores de localizaciones no periféricas ${ }^{19}$.

\section{Metástasis hepáticas irresecables (MHI) potencialmente convertibles a resecables}

\section{Tratamiento sistémico}

Uso de quimioterapia neoadyuvante: 10-30\% de los pacientes que son considerados irresecables inicialmente, posterior al tratamiento con quimioterapia se "convierten" en resecables. El desarrollo de la terapia sistémica y el mejor conocimiento de la biología del CCR, ha permitido seleccionar mejor los casos de aquellos pacientes que potencialmente se convertirán. La asociación de esquemas de citotóxicos (FOLFOX, FOLFIRI) y anticuerpos monoclonales (bevacizumab) han logrado conversión de hasta $40 \%$ de MHI, así como se ha asociado la mutación KRAS y presencia de protooncogen BRAF como factores de mal pronóstico ${ }^{4}$.

\section{Terapias regionales}

Terapia de infusión arterial hepática (quimioterapia): La vascularización de las MH es principalmente arterial (parénquima es portal) y los fármacos citotóxicos tienen un metabolismo importante de primer paso, la administración selectiva de quimioterapia en la circulación hepática permite administrar dosis más altas con menor toxicidad sistémica. Existe hasta un $50 \%$ de conversión de MHI con esta técnica ${ }^{9,19}$.

TACE (transarterial chemoembolization): Es un tratamiento mínimamente invasivo guiado por imágenes que enfrenta el tumor de dos formas: inyección arterial de quimioterapia y embolización selectiva de arteria hepática provocando necrosis del tumor. Esta terapia ha sido aceptada para el manejo de MHI sin compromiso vascular con intención de conversión ${ }^{9,19}$.

Radioembolización arterial con Y-90: Si bien la RDT locorregional no tiene beneficio en MHCCR, se ha demostrado la utilidad de radioterapia dirigida como tratamiento paliativo o de salvataje para convertir MHI, luego de que han fallado la primera y segunda línea de quimioterapia. Aun en desarrollo ${ }^{19}$.

\section{Estrategias para aumentar el RHF}

Embolización portal y ligadura portal: Inducen atrofia en los segmentos que serán resecados e hipertrofia del RHF. Esto se produce 4 a 6 semanas postprocedimiento. Tiene la limitación de que $20-30 \%$ de los pacientes sufren progresión de la enfermedad en este intervalo y no son resecados postprocedimiento ${ }^{9,19}$.

Hepatectomía en dos tiempos: Se realiza en lesiones multifocales bilobares. Consiste en 2 etapas, primero se resecan metástasis pequeñas en el RHF y en forma concomitante ligadura portal o embolización. La segunda etapa es posterior al periodo de hipertrofia, en que se reseca el lóbulo embolizado ${ }^{9,19}$. 
ALPPS “Associating Liver Partition and Portal Vein Ligation for Staged Hepatectomy”: El objetivo de esta técnica es aumentar el RHF en menos tiempo para evitar la progresión de la enfermedad durante el tiempo de espera. La primera etapa consiste en la transección entre el hígado que se resecará y el RHF, metastasectomía de las lesiones pequeñas en el RHF y la ligadura portal para minimizar el flujo entre los lóbulos. En intervalo de espera es de 7 a 10 días en que existe una rápida hipertrofia del remanente, posterior a este período de realiza la resección del hígado sin flujo portal ${ }^{17,19}$.

\section{Metástasis hepáticas irresecables, no convertibles a resecables}

La resección de la $\mathrm{MH}$ se considera el único tratamiento curativo para los pacientes en etapa IV, pero solo un $20 \%$ logran criterios de resección. La QMT paliativa es la única opción para los pacientes con $\mathrm{MHI}$, con $\mathrm{SV}$ promedio de 2 años y a 5 años de $10 \%$.

El transplante hepático $(\mathrm{TH})$ se considera el tratamiento estándar en otras neoplasias no resecables como hepatocarcinomas (HCC) y metástasis de tumores neuroendocrinos de bajo grado ${ }^{8}$. Por lo mismo, en metástasis colorrectales irresecables, el TH surge como alternativa de curación en pacientes seleccionados. Las primeras experiencias reportadas en los inicios de los 90 tuvieron sobrevida a 5 años menor al $20 \%$, por lo que se abandonó. Sin embargo, muchos de esos pacientes murieron por complicaciones relacionadas con el trasplante y no de la neoplasia.

Para poder tener éxito la clave es la selección. Tosso y col, asociaron mejores resultados en pacientes que tienen enfermedad metastásica estable, que se refleja en un intervalo largo entre la aparición de las metástasis y el $\mathrm{TH}^{22}$.

En Noruega, el estudio prospectivo SECA-I el 2013, publicó los resultados de 21 pacientes con MHCCR irresecables con $\mathrm{TH}$, obteniendo una sobrevida global (Kaplan Meier) a 5 años de $60 \%$, a pesar de que todos los pacientes tuvieron recurrencia. El mismo grupo publicó recientemente una segunda fase SECA II-2019, con 14 pacientes seleccionados, reportando una sobrevida a 5 años de $83 \%$, con seguimiento promedio de 36 meses. Cuatro pacientes no tuvieron recurrencia. Comparados con los pacientes con HCC (criterios de Milan y extendidos), las MHCCR tienen más recidiva, pero aún así la sobrevida global es mayor ${ }^{23}$.

El principal desafío para establecer el TH como una opción en pacientes con metástasis colorrectal irresecable, está relacionado con la escasez donantes cadavéricos. Muchos países tienen un largo tiempo de espera para el TH y alta mortalidad asociada a la lista de espera. Añadir una nueva indicación con muchos pacientes potencialmente candidatos, haría más crítica la situación. Se han planteado diferentes estrategias para obtener injertos para este grupo de pacientes, que al no tener hipertensión portal ni enfermedad hepática subyacente avanzada, se pueden beneficiar de hígados con criterio extendido o volumétricamente menores provenientes, por ejemplo, de donantes vivos o hepatectomías parciales en donante cadavérico. Innovadoras técnicas se han publicado, las técnicas RAPID (Noruega), en que injerto proviene de un donante cadavérico y LD-RAPID descrita por Königsrainer y en el estudio LIVER-T(W)OHEAL, donde el injerto se obtuvo de un donante vivo, consisten en dos etapas: en la primera se realiza una hepatectomía izquierda en el receptor y el trasplante de los segmentos laterales izquierdos (II y III), asociado a la ligadura portal derecha. En un segundo tiempo, 2 semanas después basados en la técnica ALPPS, cuando se ha observado un crecimiento óptimo del injerto se realiza la hepatectomía derecha ${ }^{24-26}$.

El uso de donantes vivos de hígado, aumenta claramente la oferta de órganos sin perjudicar a los pacientes enlistados. Este recurso puede ser utilizado para el tratamiento de metástasis colorrectales irresecables con el fin de lograr el preciado R0. Experiencias iniciales son alentadoras en término de lograr buenas sobrevidas, sin embargo, se requieren estudios prospectivos de alta calidad que confirmen esta tendencia, así como también mejorar los criterios de selección de pacientes para evitar las elevadas cifras de recurrencia. La técnica de donante vivo adulto-adulto por otra parte requiere un alto nivel de entrenamiento y experiencia dado la complejidad técnica y el riesgo de serias complicaciones asociadas a la cirugía de los donantes (personas completamente sanas). Sin embargo, en centros especializados se logran cifras seguras de morbimortalidad con donantes vivos que hacen aceptable esta opción para el tratamiento con intención curativa de pacientes con metástasis colorrectales irresecables que cumplan criterios establecidos.

En conclusión, el tratamiento actual de las metástasis hepáticas de origen colorrectal, debe ser discutido en un equipo multidisciplinario que incluya radiólogos intervencionales, oncólogos, gastroenterólogos y cirujanos de hígado y colorrectal. Dado la variedad de estrategias y técnicas 
conducentes a lograr R0 en la enfermedad metastásica del hígado, es fundamental no descartar a los pacientes potenciales candidatos a resección sin antes haber pasado por múltiples evaluaciones de los distintos especialistas. El trasplante de hígado aparece como una alternativa más para lograr este objetivo. Para ello debe existir la posibilidad de donante vivo y cumplir estrictamente con los criterios establecidos.

\section{Responsabilidades éticas}

Protección de personas y animales. Los autores declaran que para esta investigación no se han realizado experimentos en seres humanos ni en animales.

Confidencialidad de los datos. Los autores declaran que en este artículo no aparecen datos de pacientes.

Conflictos de interés: no hay.

\section{Referencias}

1. Allemani C, Matsuda T, Di Carlo V, Harewood R, Matz M, Nikšić M, et al. Global surveillance of trends in cancer survival 2000-14 (CONCORD-3): analysis of individual records for 37513025 patients diagnosed with one of 18 cancers from 322 populationbased registries in 71 countries. Lancet 2018;391(10125):1023-75.

2. GLOBOCAN 2018.

3. Ministerio de Salud Chile. 2019. Plan nacional de cáncer 2018-2028.

4. Lee R, Cardona K, Russell M. Historical perspective: Two decades of progress in treating metastatic colorectal cancer. J Surg Oncol. 2019;119:549-63.

5. Adam R, de Gramont A, Figueras J, Kokudo N, Kunstlinger F, Loyer E, et al. Managing synchronous liver metastases from colorectal cancer: A multidisciplinary international consensus. Cancer Treat. Rev. 2015;41:729-41.

6. Fong Y, Fortner J, Sun R, Brennan M, Blumgart L. Clinical score for predicting recurrence after hepatic resection for metastatic colorectal cancer: Analysis of 1001 consecutive cases. Ann Surg. 1999;230:309-21.

7. Norero E, Jarufe N, Butte J, Norero B, Duarte I, Torres J, et al. Resultados del tratamiento quirúrgico de las metástasis hepáticas por cáncer colorrectal. Rev Med Chile 2009;137:487-96.

8. Spolverato G, Ejaz A, Azad N, Pawlik T. Surgery for colorectal liver metastases: The evolution of determining prognosis. World J Gastrointest Oncol. 2013;5:207.

9. Chakedis J, Squires M, Beal E, Hughes T, Lewis H, Paredes A, et al. Update on current problems in colorectal liver metastasis. Curr Probl Surg. 2017;54:554602.
10. Chow F, Chok K. Colorectal liver metastases: An update on multidisciplinary approach. World J Hepatol. 2019;11:150-72.

11. Chapman W, Hoff P, Strasberg S. Selection of Patients for Resection of Hepatic Colorectal Metastases: Expert Consensus Statement by Charnsangavej, et al. Ann Surg Oncol. 2006;13:1269-70.

12. Adams R, Aloia T, Loyer E, Pawlik T, Taouli B, Vauthey J. Selection for hepatic resection of colorectal liver metastases: Expert consensus statement. HPB 2013;15:91-103.

13. Cieslak K, Huisman F, Bais T, Bennink $\mathrm{R}$, van Lienden K, Verheij J, et al. Future remnant liver function as predictive factor for the hypertrophy response after portal vein embolization. Surg. (United States) 2017;162:37-47.

14. Pawlik T, Scoggins C, Zorzi D, Abdalla E, Andres A, Eng C, et al. Effect of surgical margin status on survival and site of recurrence after hepatic resection for colorectal metastases. Ann Surg. 2005;241:715-24

15. Sadot E, Koerkamp B, Leal J, Shia J, Gonen M, Allen P, et al. Resection margin and survival in 2368 patients undergoing hepatic resection for metastatic colorectal cancer surgical technique or biologic surrogate? Ann Surg. 2015;262:476-83.

16. Adam R, Miller R, Pitombo M, Wicherts D, de Haas R, Bitsakou G, et al. Twostage Hepatectomy Approach for Initially Unresectable Colorectal Hepatic Metastases. Surg Oncol Clin N Am. 2007;16:525-36.

17. Serrano $P, G u C$, Husien M, Jalink D, Martel G, Tsang M. Effect of PET-CT on disease recurrence and its management in patients with potentially resectable colorectal cancer liver metastases: The long-term results of a randomized controlled trial (PET-CT Imaging prior to liver resection for colorectal adenocarcinoma metastases). J Clin Oncol. 2018;36:15suppl:3527.

18. Memeo R, de Blasi V, Adam R, Goéré D, Azoulay D, Ayav A, et al. Parenchymalsparing hepatectomies (PSH) for bilobar colorectal liver metastases are associated with a lower morbidity and similar oncological results: a propensity score matching analysis. HPB 2016;18:781-90.

19. Jarufe N. Impacto del tipo de resección (anatómico y no anatómico) en los resultados de la cirugía hepática para el tratamiento de las metástasis colorrectales. Rev Chil Cir. 2009;61:148-57.

20. De Andrade J, Warner S, Fong Y. Treatment of metastatic colorectal cancer: innovations in surgical techniques. J Surg Oncol. 2019;119:653-9.

21. Lykoudis P, O’Reilly D, Nastos K, Fusai G. Systematic review of surgical management of synchronous colorectal liver metastases. Br J Surg. 2014;101:60512.

22. Fretland A, Dagenborg V, Bjørnelv G, Kazaryan A, Kristiansen R, Fagerland M, et al. Laparoscopic Versus Open Resection for Colorectal Liver Metastases. Ann Surg. 2018;267:199-20.

23. Toso C, Pinto Marques H, Andres A, Castro Sousa F, Adam R, Kalil A, et al. Liver transplantation for colorectal liver metastasis: Survival without recurrence can be achieved. Liver Transpl. 2017;23:1073-6

24. Dueland S, Syversveen T, Solheim J, Solberg S, Grut H, Bjørnbeth B, et al. Survival Following Liver Transplantation for Patients With Nonresectable Liver-only Colorectal Metastases. Ann Surg. 2019 Jun 7. doi:10.1097/ SLA.0000000000003404. [Epub ahead of print]. 
CIRUGía AL díA

25. Line P, Hagness M, Berstad A, Foss A, Dueland S. A novel concept for partial liver transplantation in nonresectable colorectal liver metastases: The RAPID concept. Ann Surg. 2015;262:e5-e9.

26. Königsrainer A, Templin S, Capobianco I, Königsrainer I, Bitzer M, Zender L, et al. Paradigm Shift in the Management of Irresectable Colorectal Liver Metastases. Ann Surg. 2019;270:327-32.

27. Rauchfuß F, Nadalin S, Königsrainer A, Settmacher U. Living donor liver transplantation with two-stage hepatectomy for patients with isolated, irresectable colorectal liver - The LIVERT(W)O-HEAL study 11 Medical and Health Sciences 1112 Oncology and Carcinogenesis 11 Medical and Health Sciences 1103 Clinical Sciences. World J Surg Oncol. 2019;17:11. doi: 10.1186/ s12957-018-1549-5. 